\title{
ene
}

\section{Guía RNAO de Buenas Prácticas Clínicas en lactancia Materna - FOMENTO Y APOYO AL INICIO, LA EXCLUSIVIDAD Y LA CONTINUACIÓN DE LA LACTANCIA MATERNA PARA RECIÉN NACIDOS, LACTANTES Y NiÑos PEQUeÑos}

María del Carmen Ariza-Salamanca

MATRONA-GESTORA DE CUIDADOS DEL ÁREA DE PARITORIO 


\section{RESUMEN}

\section{Referencia del documento de práctica clínica basada en la evidencia:}

Registred Nurses Association of Ontario. Lactancia materna. Guía de buenas prácticas en enfermería. Versión española traducida por investen-isciii de: Breastfeeding Best Practice Guidlines for nurses. Toronto, Canadá: RNAO; 2018. Disponible en:

https://rnao.ca/sites/rnao-ca/files/bpg/translations/BPG_Lactancia_Materna.pdf

\section{Introducción}

La lactancia materna exclusiva hasta los seis meses (durante los cuales el lactante no recibe ningún otro líquido o sólido, excepto vitaminas en forma de gotas o jarabe, suplementos minerales o medicamentos que se le hayan prescrito) es la alimentación más favorable en los lactantes para promover unos resultados en salud favorables para neonatos, lactantes y niños pequeños.

La Guía de RNAO "Lactancia materna: buenas prácticas en enfermería" tiene como objetivo que las enfermeras, el equipo interprofesional y los pares la empleen para fomentar el inicio de la lactancia materna, su exclusividad y su continuidad, durante dos años o más, junto con la alimentación complementaria.

Además, a nivel nacional, en el año 2017 se editó la Guía de Práctica Cínica (GPC) sobre lactancia materna del Ministerio de Sanidad, Servicios Sociales e Igualdad que trata de identificar las condiciones que favorecen el inicio, la instauración y el mantenimiento de la lactancia materna e incluye recomendaciones basadas en la evidencia científica que ayuden a los profesionales sanitarios a dar respuesta a los problemas que se encuentran las madres que quieren amamantar a sus hijos.

Ambas recomiendan la realización del contacto piel con piel (CPP) madre - recién nacido y reconocen que se trata de una estrategia clave para facilitar la lactancia materna.

\section{Objetivo}

Analizar la evidencia científica disponible en relación a la buena práctica clínica: "realización del contacto piel con piel madre - recién nacido", que corresponde a la recomendación 2.1 de la Guía de RNAO de Lactancia Materna:

- Facilitar el contacto piel con piel entre la díada lactante inmediatamente tras el parto cuando esté clínicamente estable. 
- $\quad$ Nivel de evidencia para el resumen: la.

- Calidad de Evidencia para el Resumen: Alta=2; Moderada=1.

\section{Métodos, fuentes de datos}

Revisión de la Guía RNAO "Lactancia materna: buenas prácticas en enfermería"en comparación con otras evidencias (GPC sobre lactancia materna del Sistema Nacional de Salud (SNS) y otras, revisiones sistemáticas y artículos concretos referenciados en las mismas).

En la Guía RNAO para valorar la calidad de la evidencia, se utilizó la herramienta AMSTAR para las revisiones y el Agree II para las guías. El nivel de evidencia de la recomendación seleccionada es la, es decir, se corresponde con evidencia obtenida del metaanálisis o de revisiones sistemáticas de ensayos controlados aleatorizados, y/o la síntesis de diversos estudios procedentes principalmente de la investigación cuantitativa.

El CPP es uno de los principios de los "Diez pasos para una lactancia materna exitosa" de la OMS/UNICEF, que además fomenta el inicio y la exclusividad de la lactancia materna.

\section{CPP madre-recién nacido y lactancia materna}

EI CPP madre-recién nacido ha demostrado tener una asociación estadísticamente significativa con la lactancia materna exclusiva, cuyo efecto se mantiene a los 3 y 6 meses, en comparación con los recién nacidos que no realizan CPP ó lo realizan con el otro progenitor. EI CPP padre-recién nacido no ha demostrado beneficios en materia de lactancia materna, comparado con la no realización del mismo. Por ello, dicha recomendación ha demostrado calidad de evidencia, en su asociación estadísticamente significativa con la lactancia materna exclusiva, en la díada lactante clínicamente estable, es decir:

- Neonato que nace a término o poco antes de término y sano.

- Peso neonatal mayor a 2500 gramos.

- Neonato que no muestra signos de estrés respiratorio o cardiaco.

- Constantes vitales y pérdida de sangre postparto de la persona lactante dentro de los límites normales.

EI CPP madre-recién nacido se asocia a menos problemas en la lactancia materna, incluyendo de postura y agarre o de insuficiencia de leche percibi- 
da. Además, si se inicia inmediatamente después del parto se evidencian mejores niveles de confianza en la lactancia materna, mayor sensación de control en la lactancia, mayor capacidad de respuesta ante las señales de alimentación del lactante, mayor vinculación y mayor apego. Los neonatos presentan mayores probabilidades de éxito en su primer intento de amamantamiento, medido según su disposición para alimentarse, reflejo de búsqueda u hociqueo, agarre y patrón de succión. Sus niveles de glucosa medios fueron más altos tras el contacto piel con piel.

Si analizamos la realización del CPP madre-recién nacido y la lactancia materna y clasificamos según tipo de parto, tal y como se recoge en la Guía de Práctica Clínica sobre Lactancia Materna en el Sistema Nacional de Salud (2017), no se encuentra asociación estadísticamente significativa entre el contacto precoz piel con piel madre-recién nacido en cesáreas y la lactancia materna exclusiva exclusiva al alta hospitalaria. Sin embargo, el contacto precoz piel con piel madre-recién nacido en cesáreas facilita el inicio temprano de la lactancia materna, mejora la satisfacción materna, reduce el dolor materno y facilita la estabilización fisiológica de la madre y el recién nacido.

\section{Beneficios y daños del CPP madre-recién nacido}

Raramente se produce un evento catastrófico asociado al CPP precoz, conocido como colapso posnatal repentino inesperado (SUPC, por sus siglas en ingles). La incidencia del SUPC varía de 2.6 a 5.0 por cada 100000 nacidos vivos y los índices de mortalidad son de 0 a 1.1 por 100000 nacidos vivos.

La evidencia sugiere realizar valoraciones periódicas para reducir el riesgo de colapso posnatal repentino inesperado:

- Postura: la boca y la nariz deben quedar visibles y sin obstrucciones.

- Color de la piel.

- Frecuencia y esfuerzo respiratorios.

- $\quad$ Niveles de saturación de oxígeno.

- Temperatura axilar. 


\section{COMENTARIO}

En notas y prácticas en relación al CPP madre-recién nacido la Guía RNAO recoge que no hay evidencia suficiente sobre el momento óptimo del CPP y el inicio de la lactancia materna, tampoco que determine la duración óptima del mismo. Para complementar alguno de estos aspectos que ayuden a homogeneizar la práctica asistencial, se consultaron otras evidencias.

\section{GPC sobre Lactancia Materna del SNS (2017)}

Se recogen dos recomendaciones con un nivel de evidencia fuerte en relación al contacto piel con piel inmediato e ininterrumpido postparto:

Se recomienda realizar el contacto piel con piel inmediato e ininterrumpido tras el parto, colocando al recién nacido en decúbito prono, desnudo, con la cabeza ladeada y en contacto piel con piel sobre el abdomen y pecho desnudos de la madre durante los primeros 120 minutos tras el nacimiento. Se recomienda secar suavemente la cabeza y espalda del recién nacido, pero no sus manos. Para evitar la pérdida de calor se recomienda cubrirle con una manta precalentada y ponerle un gorro. El estado de la madre y del recién nacido deben ser supervisados durante ese tiempo por un acompañante correctamente informado o por un profesional sanitario.

En recién nacidos por cesárea, se recomienda también realizar contacto piel con piel inmediato e ininterrumpido tras la extracción fetal salvo que la situación del recién nacido o la madre lo impidan. Los cuidados que sean necesarios se pueden realizar con el recién nacido sobre el pecho de la madre.

En la GPC del SNS la calidad de la evidencia se midió mediante el sistema GRADE. La recomendación elegida posee un nivel de evidencia fuerte, es decir, corresponde con evidencia científica obtenida en estudios observaciones sin factores de confusión y que han mostrado una fuerte asociación ( $R R>2$ $0<0.5)$.

GPC NICE (2006) e iHAN (Iniciativa para la Humanización de la Asistencia al Nacimiento y la Lactancia,2016)

El CPP tras el parto tiene beneficios sobre la lactancia materna, y recomienda que las mujeres hagan CPP con su recién nacido tan pronto como sea posible tras el parto, evitando la separación de la madre y el recién nacido durante la primera hora de vida para realizar procedimientos rutinarios.

Guía de la Perinatal Services British Columbia (PSBC) 
Recoge que durante la primera hora tras el parto los recién nacidos están alerta y que su reflejo de succión es intenso, por lo que recomienda colocar al recién nacido en CPP con la madre tras el nacimiento para que tenga acceso directo al pecho, manteniendo el CPP hasta la finalización de la primera toma.

\section{GPC del Parto Normal del SNS (2010)}

Recomienda que las mujeres mantengan al recién nacido en CPP inmediatamente después del nacimiento, evitando la separación de madre y recién nacido dentro de la primera hora de vida y hasta que haya finalizado la primera toma.

\section{Revisión Cochrane de Moore et al. (2012) sobre el CPP madre-recién naci- do}

En relación a los nacimientos mediante cesárea se extrae que los beneficios del CPP madre-recién nacido en el inicio temprano de la lactancia materna son iguales independientemente de la modalidad de parto, en cambio, si influye el momento de inicio del mismo, presentando mayores beneficios si se inicia en los primeros 30 minutos.

\section{CPP madre-recién nacido como política sanitaria basada en la evidencia}

Como se ha mencionado anteriormente la GPC de Lactancia Materna del SNS clasifica la calidad de la evidencia mediante el sistema GRADE. Las implicaciones de la fuerza de recomendación en el sistema GRADE dependen de si se trata de una recomendación fuerte o débil. La recomendación de la realización del CPP madre-recién nacido es fuerte, lo que tiene las siguientes implicaciones:

- Para pacientes: la inmensa mayoría de las personas estarían de acuerdo con la acción recomendada y únicamente una pequeña parte no lo estarían.

- Para clínicos: la mayoría de los pacientes deberían recibir la intervención recomendada.

- Para gestores/planificadores: la recomendación puede ser adoptada como política sanitaria en la mayoría de las situaciones.

En la Estrategia de Atención al Parto Normal del SNS se recoge como indicador la realización del CPP madre - recién nacido y su valor óptimo se cifra $\geq 80 \%$. Este estándar es más asequible en partos vía vaginal que en nacimien- 
tos mediante cesárea. EI CPP madre-recién nacido tras una cesárea es una práctica que se ha de promocionar ya que no está suficientemente implantada en los hospitales y los profesionales sanitarios aún muestran reticencias. Son diversos los estudios que han planteado las resistencias de los profesionales sanitarios, se observó que dichas resistencias disminuyen con la formación de los mismos.

En resumen, de acuerdo a la evidencia científica disponible el CPP madre-recién nacido en la díada lactante estable debería ser adoptado como política sanitaria en la mayoría de las situaciones, con independencia del tipo de parto; ya que ha demostrado tener una asociación estadísticamente significativa con la Lactancia Materna Exclusiva (LME), fundamentalmente cuando se inicia en los primeros 30 minutos tras el nacimiento y se mantiene hasta, al menos, finalizada la primera toma, siendo necesarios estudios que analicen el momento y duración óptimos.

\section{REFERENCIAS}

1. Registred Nurses Association of Ontario. Lactancia materna. Guía de buenas prácticas en enfermería. Versión española traducida por investen-iscii de: Breastfeeding Best Practice Guidlines for nurses. Toronto, Canadá: RNAO; 2018. Disponible en: https://rnao.ca/sites/rnao-ca/files/bpg/translations/BPG_Lactancia_Materna.pdf

2. Grupo de trabajo de la Guía de Práctica Clínica sobre lactancia materna. Guía de Práctica Clínica sobre lactancia materna. Ministerio de Sanidad, Servicios Sociales e Igualdad; Agencia de Evaluación de Tecnologías Sanitarias del País Vasco-OSTEBA, 2017. Guías de Práctica Clínica en el SNS.

3. Moore ER, Anderson GC, Bergman N, et al. Early skin-to-skin contact for mothers and their healthy newborn infants. Cochrane Database Cyst. Rev. 2012(5): CD003519.

4. Minsterio de Sanidad y Consumo; Observatorio de Salud de la Mujer y del Sistema Nacional de Salud.Es trategia de atención al parto normal en el Sistema Nacional de Salud.[monografía de Internet]. Madrid: Ministerio de Sanidad y Consumo; 2008 [acceso septiembre de 2020]. Disponible en: www.msc.es/organización/ sns/planCalidadSNS/pf/excencia/atenciónParto/ estrategiaPartoEnero2008.pdf. 